\title{
The ZigBee Based Wireless Sensor and Actor Network in Intelligent Space Oriented to Home Service Robot
}

\author{
Fei Lu, Guo-Hui Tian* \\ School of Control Science and Engineering, Shandong University, Jinan, China \\ Email: \{lawyerlf, "g.h.tian\}@sdu.edu.cn
}

Received March 23, 2012; revised April 18, 2012; accepted April 25, 2012

\begin{abstract}
In the study of intelligent space oriented to home service robot, an important technology is how to construct an communication network which has the characters of high reliability and easy building. In this paper, based on the characteristics of ZigBee protocol, ZigBee technology is used to construct a wireless sensor and actor network. Several intelligent services based on ZigBee wireless sensor and actor network are shown to certify the reliability of this communication network. ZigBee wireless sensor and actor network builds an information bridge for the components in the intelligent space, the spatially distributed devices are connected together seamlessly. With this network, robot can share the mass information in the intelligent space and improve its performance with "light-packs", devices in intelligent space, such as lamp, curtain can be controlled autonomously.
\end{abstract}

Keywords: Intelligent Space; Home Service Robot; Wireless Sensor and Actor Network; ZigBee Technology

\section{Introduction}

The concept of ubiquitous computing is proposed by Mark Weiser in 1991, it is a new computing paradigm [1]. Currently, there are some research fields under ubiquitous computing, such as: intelligent space, context-aware computing, nomadic computing [2]. In recent decade, as the aging problem becomes increasingly serious in many countries and in order to improve the life quality of eldly persons and persons with physical disabilities, many attention has been put into the field of intelligent space oriented to home service robot [3]. Intelligent space becomes a platform of ubiquitous computing.

An intelligent space is an area such as a room, a corridor or a street that is equipped with sensors (CCD cameras, microphones etc.), actuators (display, speaker etc.), information database, communication devices and computational ability [4]. The intelligent space can continuously monitor what is happening in it, can communicate with its inhabitants, can make related inference and decisions and act on these decisions. It can be used to enhance the quality of people's the everyday life in it.

Wireless sensor network, the key point in information acquisition and command transmission, is one of the most important technology for intelligent space. It has attracted substantial attention. Distributed intelligent network device proposed by Hashimoto is one of the earliest network to implement an intelligent space using network

\footnotetext{
*Corresponding author.
}

devices [4]. Baeg initiated a smart home environment project for light-weight service robots to provide reliable services through the wireless sensor networks [5]. Luo and his colleagues described a prototypical configuration for networked robot systems [6]. Suh suggested a new intelligent home control system based on wireless sensor/ actuator network, which divided and assigned various home network tasks to appropriate components [7]. Yu implemented a ubiquitous robotic space with sensor network based on ZigBee protocol [8]. Wireless sensor and actor networks is a new network module. It is a distributed sensor and control system which is developed from the wireless sensor network by add many kinds of actuators. It can finish the distributed information collection and perform scheduled tasks [9]. How to build a communication network which is high reliable and easy to be built is very important technology in the intelligent space oriented to home service robot, it is the basis for the robot to provide the intelligent service for the human. In the intelligent space oriented to home service robot, since ZigBee technology has the characters of lower speed, lower power and less complexity, it is adopted to build wireless sensor and actor network to transfer the environmental sensor data, the intelligent space commands and their feedback, so that the intelligent space and service robots can be closely connected.

This paper is organized as follows. Section 2 described the heterogeneous network module in intelligent space oritened to home service robot; in Section 3, ZigBee 
technology and the frame of ZigBee protocol were introduced; Section 4 introduced the intelligent home service in intelligent space based on ZigBee wireless sensor and actor network; Section 5 concluded our work and presented the future direction of our research.

\section{Heterogeneous Networks in Intelligent Space}

The perception and service capabilities of service robot have been greatly extended by combining with the intelligent space technology. Intelligent space can provide mass information in the home environment. In order to share these information, a heterogeneous networks is built. It includes several networks, such as wireless sensor and actor networks, local networks, Internet, GPRS. The heterogeneous network in intelligent space system is shown in Figure 1.

In the internal networks, the ZigBee protocol is adopted in to built wireless sensor and actor networks in intelligent space. It can be used not only to transfer the environmental sensor data to the sever, such as temperature, humidity, but also to transfer the control command decided by the sever to the intelligent device. The wireless sensor and actor networks based on ZigBee protocol is listed in Figure 2.

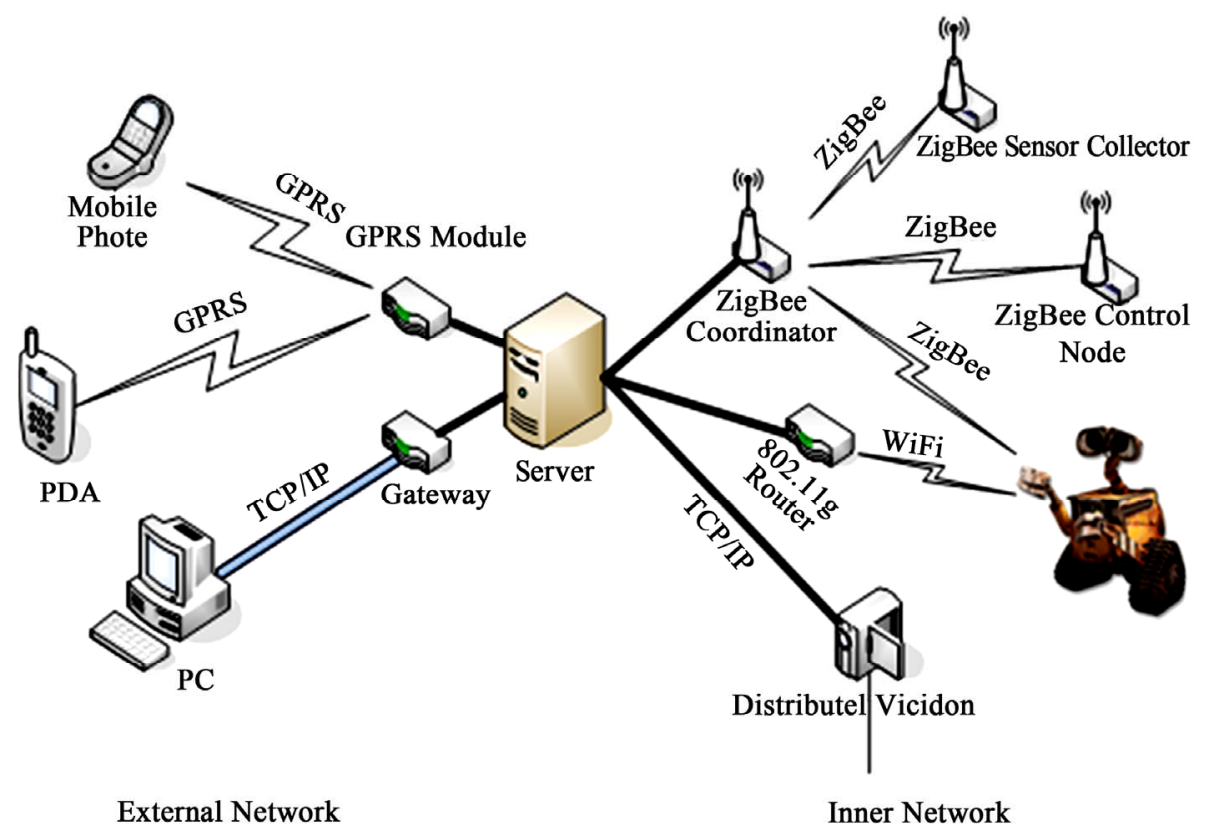

Figure 1. Heterogeneous network in intelligent space system.

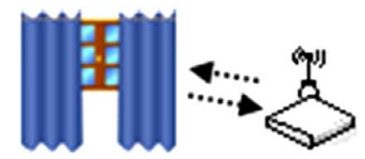

Actuator

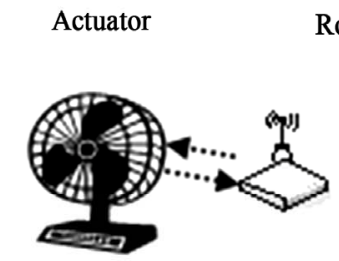

Router

Router

Router

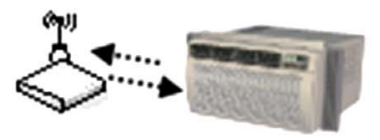

Air condition controller
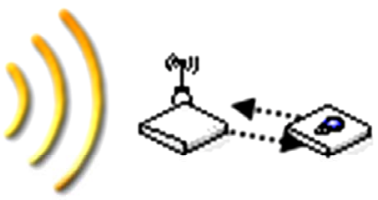

ZigBee Network

Temperature

Humidity
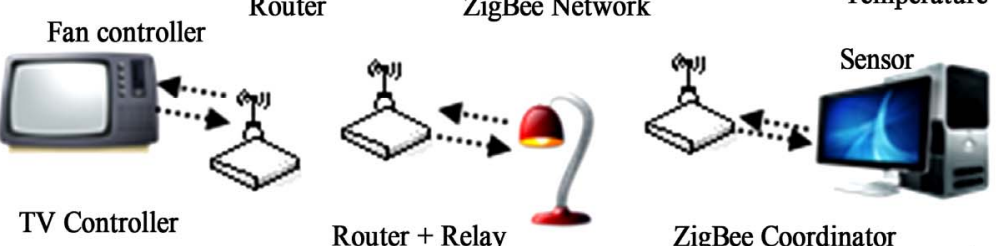

ZigBee Coordinator

Sever

Figure 2. Wireless sensor and actor networks based on ZigBee protocol. 


\section{Wireless Sensor and Actor Network Based on ZigBee Protocol}

Bluetooth (over IEEE 802.15.1), UWB (over IEEE 802.15.3), ZigBee (over IEEE 802.15.4), and Wi-Fi (over IEEE $802.11 \mathrm{a} / \mathrm{b} / \mathrm{g}$ ) are four protocol standards for short range wireless communications [10]. ZigBee is a new wireless communication technology based on wire-less standard 802.15.4. Compared with other protocol standards, ZigBee stack offers a practical application solution coupled with low rate, low cost, low energy consumption characteristics for wireless sensor network [11]. The data amount of the sensor and control commands in intelligent space oriented to home service robot are small, so the wireless sensor networks requires a little transmission rate. Based on these characteristics, ZigBee protocol is selected to construct the wireless sensor and actor network in intelligent space oriented to home service robot.

ZigBee is a novel radio frequency (RF) communications standard based on IEEE 802.15.4. It defines the application layer and network layer, utilizing the IEEE 802.15.4 standard as the communication protocols of medium access control (MAC) layer and physical (PHY) layer [12]. The structure of ZigBee protocol stack is illustrated in Figure 3.

The ZigBee wireless networks consist of three roles: one coordinator, several end devices and routers. The coordinator is a special FFD (full function device) responsible for creating and maintaining the whole ZigBee PAN (personal area network). During the network initialization phase, the coordinator scans the available radio channels to find the most suitable channel. Normally, this will be the channel with the least activity. The coor-dinator can be pre-programmed with the PAN ID (Per-sonal Area Network Identifier) or dynamically scans for existing network PAN IDs in the same frequency and generate a conflict-free PAN ID. After the coordinator's initialization phase, the coordinator waits for the requests from ZigBee devices to join the network [13]. The end device can be an FFD or RFD (reduce function device). An FFD can act as anyone of the three roles, while an RFD can only act as the end device. During the initialization, the end devices scan for available channels to identify the network distinguished by their PAN ID and requests

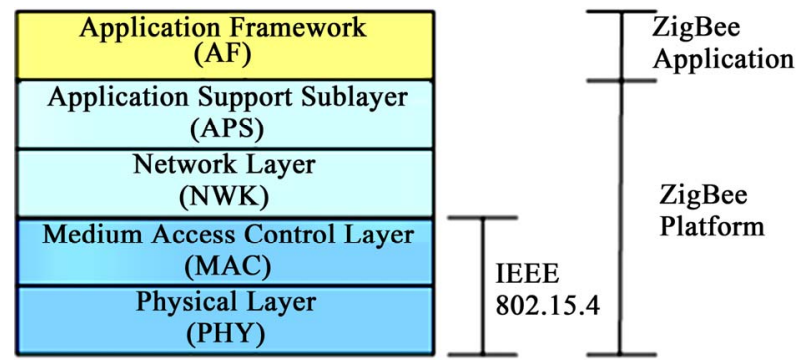

Figure 3. Structure of ZigBee protocol stack. are sent to the coordinator of the network they wish to join. Router is an optional device for ZigBee which may be needed in some special network configuration.

In addition, the topology of ZigBee wireless sensor networks includes simple star network, reliable mesh network and large scale mesh-tree network, Figure 4 illustrates the three topology structures of ZigBee wireless sensor networks. In our intelligent space oriented to home service robot, considering the easy expansion of the whole system, all of the devices in the implemented ZigBee wireless sensor networks are FFD and they form.

\section{Intelligent Home Service in Intelligent Space Based on ZigBee Wireless Sensor and Actor Network}

\subsection{The Control to the Intelligent Device}

With the help of ZigBee technology, the normal device will become an intelligent device by add ZigBee node with simple alteration. In the intelligent space system oriented to home service robot, in order to complete the collection of the sensor data and the control to the intelligent device, such as curtain, lamp, a SQL sever database of Microsoft company is adopted. Four data tables are built to save the relative data, shown as Figure 5.

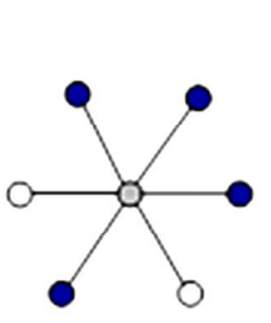

Star network ○ Coordinator

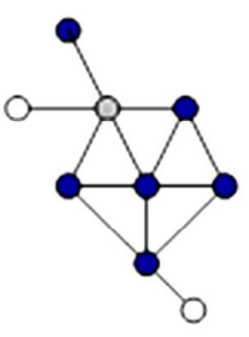

Mesh network

Router

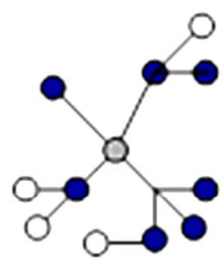

Mesh-tree network

OEnd Device
Figure 4. Topology structure of ZigBee wireless sensor network.

\begin{tabular}{|l|lll|ll|l|l|} 
S_time & & number & tem & hum & light & coul & smoke \\
\hline $2009-5-31$ & $16: 51$ & 10000000 & $29.5 C$ & $29.6 \times$ & 8.0 & Full & 0.2 \\
$2009-5-31$ & $16: 52$ & 10000000 & $29.6 C$ & $30.5 \times$ & 8.0 & Full & 0.2
\end{tabular}

sensor data table

\begin{tabular}{|c|c|c|c|}
\hline act_time & act_id & act_name & act_to_IKE \\
\hline $2009-5-6$ & & 站立 & 〈ñL। \\
\hline
\end{tabular}

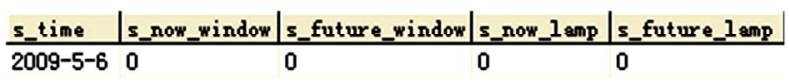

INEQ_state data table

\begin{tabular}{|l|l|l|l|}
\hline do_time & task_id & task_name & task_status \\
\hline 2009-5-6 $17: 10: 1$ & 开菑䆑 & 成功 \\
& task data table &
\end{tabular}

Figure 5. Four data tables for the control to intelligent device. 
Sensor data table is used to save all kinds of sensor data, these data are transferred to the computer by ZigBee network coordinator. Action data table is used to save the human behaviors which are detected by the related detecting program. INEQ_state data table is used to save the current status of the curtain or lamp, the default status is " 0 " which means the intelligent device is off. The actuating result of the task is saved in task data table.

Now, we will take opening the curtain as an example to introduce the control processing of server monitoring program through ZigBee wireless sensor and actor network.

The sensor, such as camera, can detect the human's action and transfer the image to the server in intelligent space. Information of human's action, such as action time are saved into the action data table.

The data-fusion program query the database continuously. When the change of the action data table is detected, the program will fuse all data information in different data table, such as the current time, current light condition of the room and make the decision about the curtain status. If the decision is not need to open the curtain, program will ignore the human's behavior, otherwise will set the "s_future_window" as " 1 " in the INEQstate data table.

When the autonomous monitoring program of the server find the "s_future_window" is " 1 " and "s_now_ window" is " 0 ", the command of open curtain will be send to ZigBee coordinator. ZigBee coordinator receives the control command through serial port from the server and send the command to the terminal node by wireless sensor network. The actuator with terminal node will actuate the respond action, open the curtain and set "s_now_window" as " 1 ”.

\subsection{Ceiling Project Control Based on ZigBee Wireless Sensor Networks}

The ceiling projector is used for robot navigation in the intelligent space oriented to home service robot. Information in intelligent space is gathered and integrated to plan a reasonable path for the mobile robot. The principle of robot navigation based on ceiling project is shown in Figure 6.

The system of robot navigation based on ceiling project is made up of server, distributed visual system, ceiling project, wireless communication system and robot. The server is the central processing unit, it decides the path planning according to the information which is provided by other units in intelligent space and transfers the path planning into the control command to the ceiling project.

The path data are sent to the controller of the ceiling projector through ZigBee wireless sensor networks, and casts a spot along the path. Then, the mobile robot fol- lows the moving spot using its onboard sensors (e.g. CCD camera). This navigation method can avoid the acquisition of sensor information and the corresponding information fusion, reduce the sensors amount equipped on mobile robot, the work of path planning is finished by the server. The robot can work with "light-packs" and improve the performance.

This paper only describes the format of data packet which is transferred by the server to the projector.

The server uses serial port to communicate with ZigBee coordinator, the data packet which sent by the server to ZigBee coordinator contains three parts is shown in Figure 7:

Part I: ZigBee node's physical address, hexadecimal, occupies 8 bytes.

Part II: Laser control information, " 1 ” means laser is on, " 0 ” means laser is off, it occupies 1 byte.

Part III: Projector control information, information format occupies 7 bytes.

Communication interface of ZigBee node is RS-232, interface of projector controller is RS-485, therefore, it is necessary to add RS-232-RS-485 converter module between ZigBee node and projector controller. After receiving the data packet broadcasted from ZigBee coordinator, ZigBee nodes begin to parse the packet. If the ZigBee physical address in the packet matches with the physical address of this node, the further data analysis will be handled. Firstly, extract the information of laser controller and decide the control of laser through the information is " 0 " or " 1 ". Secondly, extract information of projector controller, send the data of 7 bytes to the projector controller through the serial port.

Figures 8 and 9 are the robot navigation based on ceiling project and infrared sensors respectively. The yellow line is the path planned by the sever, the green line is the robot's trace. We can find the accuracy in Figure 8 is

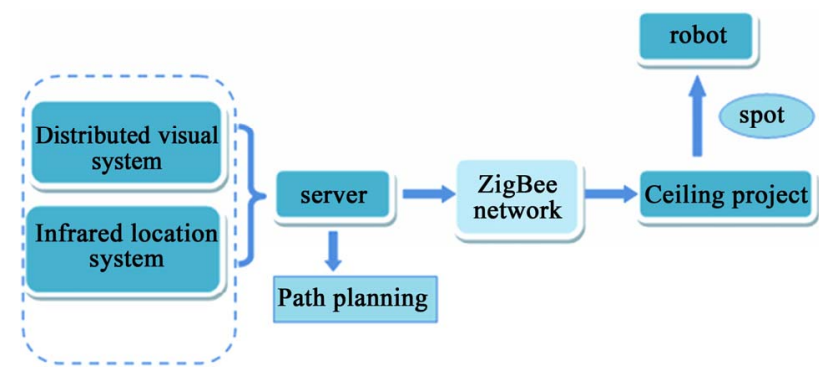

Figure 6. Principle of robot navigation based on ceiling project.

$\begin{array}{llllllllllllllll}34 & 30 & 30 & 30 & 30 & 30 & 30 & 30 & 00 & \text { A5 } & 01 & 00 & 00 & 00 & 00 & \text { A6 }\end{array}$

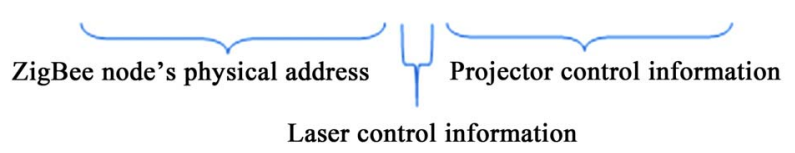

Figure 7. Data format sent by server to ZigBee coordinator. 
better than in Figure 9.

\subsection{Object Search Based on the Fusion of RFID and ZigBee}

One of the main tasks of service robot is to provide different kinds of services to persons in complex indoor environment, such as delivering water or medicine. So the robot must have the ability to search the target and recognize the target autonomously. In intelligent space, robot can finished the task successfully with the help of distributed intelligent device in the space and on-board sensor of the robot. RFID (Radio Frequency Identification) and ZigBee technology are very important for the actuating of the task [14].

The principle of object search for the robot based on the fusion of RFID and ZigBee is shown as Figure 10.

The robot will send control command to the sever in the intelligent space when it needs to search and locate an object. Sever will look for the ZigBee nodes which connect with the RFID reader and send the command to these nodes, then the ZigBee terminal node will control to turn on the RFID reader and RFID antenna. When RFID antenna finds the tag which is attached on the object, RFID reader will send the RFID tag information to the ZigBee terminal node through serial port. ZigBee terminal node will add its physical address to the RFID tag information, and transfer this information to the sever by ZigBee wireless sensor network and server will transfer this information to the robot. Robot can complete the object search according to the information. The program flowchart is show in Figure 11.

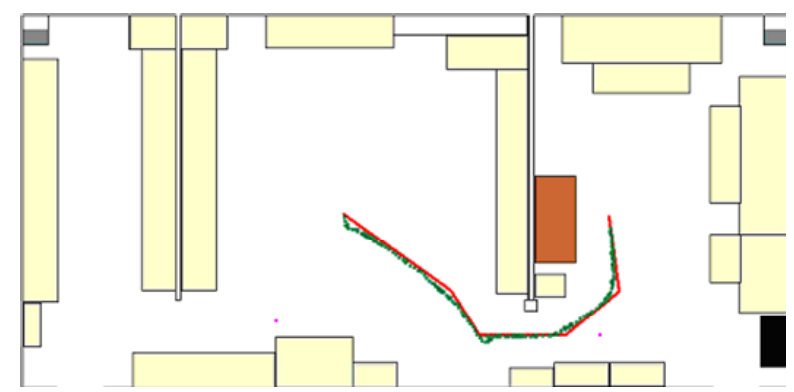

Figure 8. Robot navigation based on ceiling project.

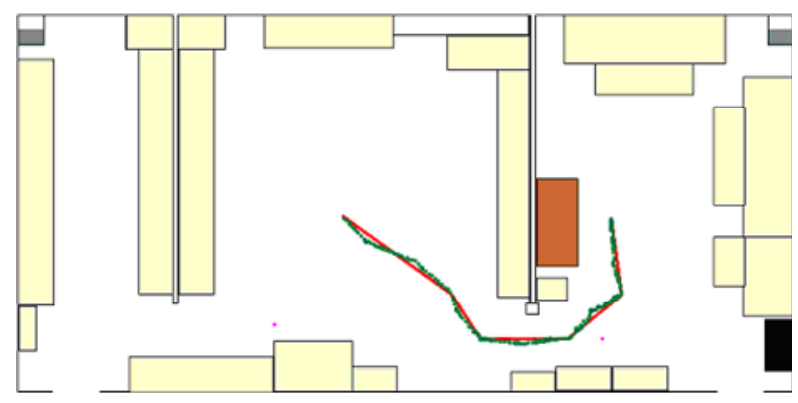

Figure 9. Robot navigation based on infrared sensors.

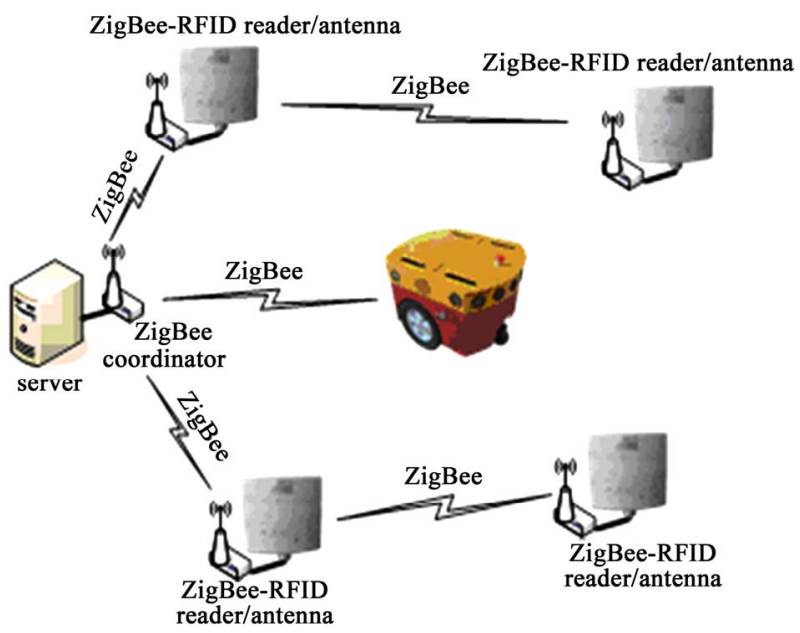

Figure 10. Object search based on ZigBee-RFID.

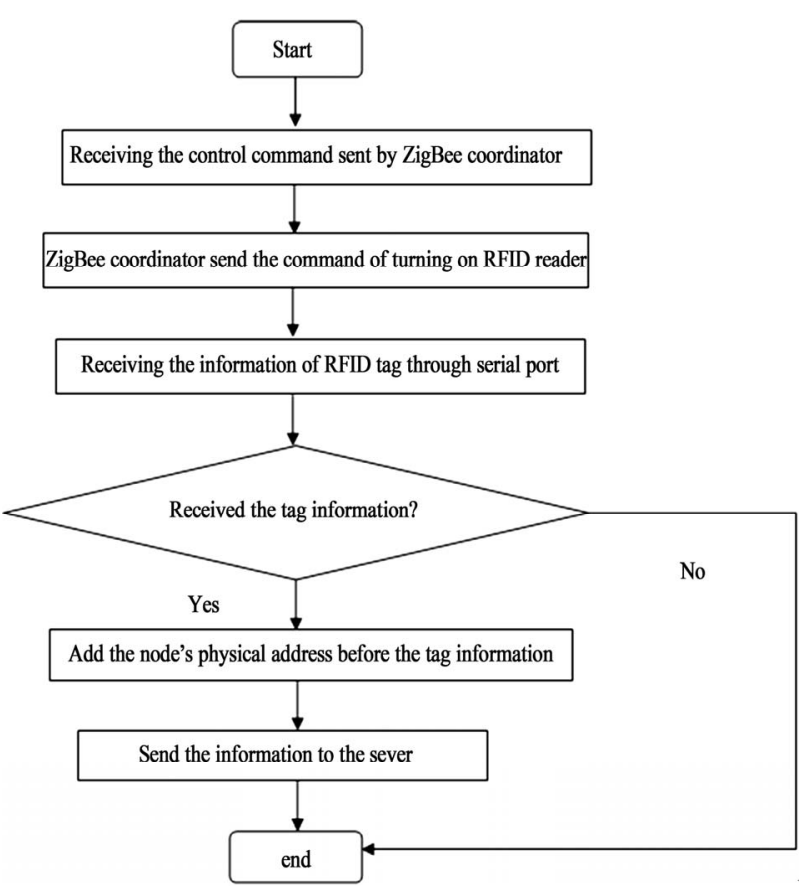

Figure 11. The flowchart of program.

\section{Conclusion and Future Work}

In this paper, the wireless sensor and actor network based on the ZigBee technology is introduced. The intelligent service based on this sensor and actor network is shown. These services certified the reliability of the network. But our study of ZigBee wireless sensor and actor network in the intelligent space oriented to home service robot is limited, there are many problems should to be studied deeply. In the future, we will do more research on this field.

\section{Acknowledgements}

The authors gratefully acknowledge the funds provided 
by National Natural Science Foundation of China under Grant 61075092, National High-tech Program of China (863 Program) under Grant 2009AA04Z220, Natural Science Foundation of Shandong Province under Grant ZR2011FM011, Independent Innovation Foundation of Shandong University under grant 2012TS077 and Independent Innovation Foundation of Shandong University under Grant 2011JC017.

\section{REFERENCES}

[1] M. Weiser, "The Computer for the Twenty-first Century," Scientific American, Vol. 265, No. 3, 1991, pp. 94-104. doi:10.1038/scientificamerican0991-94

[2] G.-Y. Xu, Y.-C. Shi and W.-K. Xie, "Pervasive/Ubiquitous Computing," Chinese Journal of Computers, Vol. 26, No. 9, 2003, pp. 1042-1050.

[3] G. H. Tian, X. L. Li, S. P. Zhao, et al., "Research and Development of Intelligent Space Technology for Home Service Robot," Journal of Shandong University: Engineering Science, Vol. 37, No. 5, 2007, pp. 53-59.

[4] J.-H. Lee and H. Hashimoto, "Intelligent Space," Proceedings of the IEEE International Conference on Intelligent Robots and Systems, Takamatsu, 30 October-5 November 2000, pp. 1358-1363.

[5] S.-H. Baeg, J.-H. Park, J. Koh, et al., "Building a Smart Home Environment for Service Robots Based on RFID and Sensor Networks," International Conference on Control, Automation and Systems, Seoul, 17-20 October 2007, pp.1078-1082. doi:10.1109/ICCAS.2007.4407059

[6] R. C. Luo, K. L. Su, S. H. Shen and K. H. Tsai, "Networked Intelligent Robots through the Internet: Issues and Opportunities," Proceedings of IEEE, Vol. 91, No. 3, 2003, pp. 371-382. doi:10.1109/JPROC.2003.809198

[7] C. S. Suh and Y.-B. Ko, "Design and Implementation of Intelligent Home Control Systems Based on Active Sen- sor Networks," IEEE Transactions on Consumer Electronics, Vol. 54, No. 3, 2008, pp. 1177-1184.

doi:10.1109/TCE.2008.4637604

[8] W. Yu, J.-Y. Lee, Y.-G. Ha, et al., "Design and Implementation of a Ubiquitous Robotic Space,” IEEE Transactions on Automation Science and Engineering, Vol. 6, No. 4, 2009, pp. 633-640. doi:10.1109/TASE.2009.2024925

[9] I. F. Akyildiz, W. Su, Y. Sankarasubramaniam, et al., "Wireless Sensor Networks: A Survey," Computer Networks, Vol. 38, No. 4, 2001, pp. 393-422. doi:10.1016/S1389-1286(01)00302-4

[10] J.-S. Lee, Y.-W. Su and C.-C. Shen, “A Comparative Study of Wireless Protocols: Bluetooth, UWB, ZigBee, and Wi-Fi," Proceedings of 33rd Annual Conference of the IEEE Industrial Electronics Society, Taipei, 5-8 November 2007, pp. 46-51.

[11] D. Y. He, "The ZigBee Wirelesss Sensor Network in Medical Care Application,” International Conference on Computer, Mechatronics, Control and Electronic Engineering, Changchun, 24-26 August 2010, pp. 497-500.

[12] E. Callaway, P. Gorday, L. Hester, et al., "Home Networking with IEEE 802.15.4 Developing Standard for Low-Rate Wireless Personal Area Networks,” IEEE Communications Magazine, Vol. 40, No. 8, 2002, pp. 70-77. doi:10.1109/MCOM.2002.1024418

[13] K. Gill, S.-H. Yang, F. Yao and X. Lu, “A ZigBee-Based Home Automation System,” IEEE Transactions on Consumer Electronics, Vol. 55, No. 2, 2009, pp. 422-430. doi:10.1109/TCE.2009.5174403

[14] Y. H. Xue, G. H. Tian, R. K. Li and H. T. Jiang, “A New Object Search and Recognition Method Based on Artificial Object Mark in Complex Indoor Environment," The 8th World Congress on Intelligent Control and Automation, Jinan, 7-8 July 2010, pp. 6648-6653. 